\title{
Extensions of a fixed point theorem of Meir and Keeler
}

\author{
Sehie Park* and Jong Sook Bae
}

\section{Introduction}

Meir and Keeler [9] established a fixed point theorem which is a remarkable generalization of the Banach contraction principle.

A selfmap $g$ of a metric space $(X, d)$ is called a weakly uniformly strict contraction or simply an $(\varepsilon, \delta)$-contraction if for each $\varepsilon>0$, there exists a $\delta>0$ such that for all $x, y \in X$,

$$
\varepsilon \leqq d(x, y)<\varepsilon+\delta \text { implies } d(g x, g y)<\varepsilon .
$$

Meir and Keeler proved that an $(\varepsilon, \delta)$-contraction $g$ of a complete metric space $X$ has a unique fixed point $\eta$ in $X$ and $\left\{g^{n} x\right\}_{n=1}^{\infty}$ converges to $\eta$ for all $x \in X$ [9]. The class of $(\varepsilon, \delta)$-contractions clearly contains the classes of (Banach) contractions and nonlinear contractions investigated by Browder [3] and by Boyd and Wong [2].

A fixed point of a selfmap $g$ of $X$ can be considered as a common fixed point of $g$ and $1_{X}$, the identity map of $X$. In certain cases, we can replace $1_{X}$ by a continuous selfmap $f$ of $X$ and consider common fixed point of $f$ and $g$. Jungck [8] adopted this idea and obtained a useful generalization of the Banach contraction principle to commuting selfmaps. More recently, Park [10] extended these facts and obtained a number of results on commuting selfmaps.

Let $f$ be a continuous selfmap of a metric space $(X, d)$ and $C_{f}$ denote the class of selfmaps $g$ of $X$ such that $f g=g f$ and $g X \subset f X$. A selfmap $g$ of $X$ is called an $(\varepsilon, \delta)-f-$ contraction if for any $\varepsilon>0$, there exists $\delta=\delta(\varepsilon)>0$ such that for all $x, y \in X$,

$$
\varepsilon \leqq d(f x, f y)<\varepsilon+\delta \text { implies } d(g x, g y)<\varepsilon,
$$

and $\left(2^{\prime}\right) g x=g y$ whenever $f x=f y$.

* This paper was written while the first author was visiting the University of California, Berkeley in 1979-80. 
In this paper we show that an $(\varepsilon, \delta)$-f-contraction $g$ in $C_{f}$ has a unique common fixed point with $f$ whenever $X$ is complete, and that this extends fixed point theorems of Meir and Keeler [9], Edelstein [5], Browder [3], Boyd and Wong [2], Jungck [8], Park [10], Jeong [7], and Chung [4]. Some related results are also obtained.

\section{Fixed point theorems}

Let $f$ and $g$ be selfmaps of a metric space $(X, d)$. Given a point $x_{0}$ in $X$, we consider a sequence $\left\{f x_{n}\right\}_{n=1}^{\infty}$ recursively given by the rule $f x_{n}=g x_{n-1}, n=1,2, \ldots$. Such a sequence is called an $f$-iteration of $x_{0}$ under $g$.

Note that for an $(\varepsilon, \delta)-f$-contraction $g$, we have

$$
d(g x, g y)<d(f x, f y) \text { for } x, y \in X, f x \neq f y .
$$

Lemma 2.1. Let $f$ be a selfmap of a metric space $X$ and $g$ be an $(\varepsilon, \delta)$-f-contraction. If there exists an $x_{0} \in X$ and an f-iteration $\left\{f x_{n}\right\}_{n=1}^{\infty}$ of $x_{0}$ under $g$, then $\left\{d\left(f x_{n}, f x_{n+1}\right) \mid n=1,2, \ldots\right\}$ is monotone decreasing to 0.

Proof. Suppose inf $\left\{d\left(f x_{n}, f x_{n+1}\right)\right\}=r$ for some $r>0$. Then by (3), we have

$$
d\left(f x_{n}, f x_{n+1}\right)=d\left(g x_{n-1}, g x_{n}\right)<d\left(f x_{n-1}, f x_{n}\right),
$$

so $\left\{d\left(f x_{n}, f x_{n+1}\right)\right\}_{n=1}^{\infty}$ is a decreasing sequence and, hence, $\lim _{n} d\left(f x_{n}, f x_{n+1}\right)=r$. By (2), there exists a $\delta>0$ such that

$$
r \leqq d(f x, f y)<r+\delta \quad \text { implies } d(g x, g y)<r .
$$

Since $\lim _{n} d\left(f x_{n}, f x_{n+1}\right)=r$, there exists a positive integer $N$ such that for every $m \geqq N$, we have

$$
r \leqq d\left(f x_{m}, f x_{m+1}\right)<r+\delta .
$$

Then for every $m \geqq N$, we have

$$
d\left(f x_{m+1}, f x_{m+2}\right)=d\left(g x_{m}, g x_{m+1}\right)<r,
$$

which contradicts (4). Therefore, we have $\lim _{n} d\left(f x_{n}, f x_{n+1}\right)=0$.

Lemma 2.2. Let $g$ be an $(\varepsilon, \delta)$-f-contraction commuting with $f$. If there exists a $\xi \in X$ such that $f \xi=g \xi$, then $f \xi$ is the unique common fixed point of $f$ and $g$.

Proof. Let $f \xi=g \xi=\eta$, and suppose $f \eta \neq \eta$. Then by (3)

$$
d(\eta, f \eta)=d(g \xi, f g \xi)=d(g \xi, g f \xi)<d(f \xi, f f \xi)=d(\eta, f \eta),
$$

which is a contradiction. Hence we have $f \eta=\eta$ and $g \eta=g f \xi=f g \xi=f \eta=\eta$. Therefore, $f \xi$ is a common fixed point of $f$ and $g$. Let $\eta^{\prime}$ be a common fixed point of $f$ 
and $g$ such that $\eta \neq \eta^{\prime}$. Then by (3)

$$
d\left(\eta, \eta^{\prime}\right)=d\left(g \eta, g \eta^{\prime}\right)<d\left(f \eta, f \eta^{\prime}\right)=d\left(\eta, \eta^{\prime}\right),
$$

which is a contradiction. Therefore $\eta$ is unique.

Now we have our main result.

Theorem 2.3. Let $f$ be a selfmap of a metric space $X$ and $g$ be an $(\varepsilon, \delta)$-f-contraction commuting with $f$. If a point $x_{0} \in X$ has an $f$-iteration $\left\{f x_{n}\right\}_{n=1}^{\infty}$ under $g$ with a cluster point $\xi \in X$ at which $f$ is continuous, then $\left\{f x_{n}\right\}$ converges to $\xi$, and $f \xi$ is the unique common fixed point of $f$ and $g$.

Proof. By Lemma 2.2, it is sufficient to show that we can find a point $\xi$ in $X$ such that $f \xi=g \xi$. If $d\left(f x_{n}, f x_{n+1}\right)=0$ for some $n$, then $f x_{n+1}=g x_{n}=f x_{n}$, and we are done. Suppose $d\left(f x_{n}, f x_{n+1}\right) \neq 0$ for every $n$. We now claim that $\left\{f x_{n}\right\}$ is a Cauchy sequence. Suppose not. Then there exists an $\varepsilon>0$ and a subsequence $\left\{f x_{n_{i}}\right\}$ of $\left\{f x_{n}\right\}$ such that

$$
d\left(f x_{n_{i}}, f x_{n_{i+1}}\right)>2 \varepsilon .
$$

By (2), there exists $0<\delta<\varepsilon$ such that

$$
\varepsilon \leqq d(f x, f y)<\varepsilon+\delta \text { implies } d(g x, g y)<\varepsilon .
$$

Since $\lim _{n} d\left(f x_{n}, f x_{n+1}\right)=0$ by Lemma 2.1 , there exists a positive integer $N$ such that for every $m \geqq N$, we have

$$
d\left(f x_{m}, f x_{m+1}\right)<\delta / 6 .
$$

Then by (5) and (6), for every $n_{i}>N$, we can find $m$ such that $n_{i}<m<n_{i+1}$ and

$$
\varepsilon+\frac{\delta}{3} \leqq d\left(f x_{n_{i}}, f x_{m}\right)<\varepsilon+\delta
$$

Then

$$
\begin{aligned}
d\left(f x_{n_{i}}, f x_{m}\right) & \leqq d\left(f x_{n_{i}}, f x_{n_{i}+1}\right)+d\left(f x_{n_{i}+1}, f x_{m+1}\right)+d\left(f x_{m+1}, f x_{m}\right) \\
& <\frac{\delta}{6}+d\left(g x_{n_{i}}, g x_{m}\right)+\frac{\delta}{6} \\
& <\varepsilon+\frac{\delta}{3},
\end{aligned}
$$

which contradicts (7), and hence $\left\{f x_{n}\right\}$ is a Cauchy sequence. Since $\left\{f x_{n}\right\}$ clusters at $\xi \in X$, it converges to $\xi$. Since $f$ is continuous at $\xi,\left\{f f x_{n}\right\}=\left\{f g x_{n-1}\right\}=\left\{g f x_{n-1}\right\}$ converges to $f \xi$.

Suppose $f f x_{m}=f f x_{m+1}=f f x_{m+2}=\ldots$ for some $m$. Then $\left\{f f x_{n}\right\}$ converges to $f f x_{m}$ and $f f x_{m}=f f x_{m+1}=f g x_{m}=g f x_{m}$. Hence $f x_{m}$ is a coincidence point of $f$ and $g$, 
and $f f x_{m}=f \xi$. Thus we are done. Suppose that we can not find an $m$ satisfying $f f x_{m}=f f x_{m+1}=\ldots$. Then for any $\varepsilon>0$, there exists an $N$ such that for every $m \geqq N$, $d\left(f f x_{m}, f \xi\right)<\varepsilon / 2$, and we can find an $n \geqq N$ such that $f f x_{n} \neq f \xi$. Then we have

$$
\begin{aligned}
d(f \xi, g \xi) & \leqq d\left(f \xi, f g x_{n}\right)+d\left(f g x_{n}, g \xi\right) \\
& =d\left(f \xi, f f x_{n+1}\right)+d\left(g f x_{n}, g \xi\right) \\
& <\varepsilon / 2+d\left(f f x_{n}, f \xi\right)<\varepsilon / 2+\varepsilon / 2=\varepsilon .
\end{aligned}
$$

Therefore $f \xi=g \xi$, and this completes our proof.

Note that if $g X \subset f X$, then every $x_{0} \in X$ has an $f$-iteration under $g$. Therefore, from Theorem 2.3, we have

Theorem 2.4. Let $f$ be a continuous selfmap of a complete metric space $X$ and $g$ be an $(\varepsilon, \delta)$-f-contraction in $C_{f}$. Then $f$ and $g$ have a unique common fixed point $\eta$ in $X$, and, for any $x_{0}$ in $X$, every $f$-iteration of $x_{0}$ under $g$ converges to some $\xi \in X$. satisfying $f \xi=\eta$.

Proof. An $f$-iteration $\left\{f x_{n}\right\}$ of $x_{0}$ under $g$ is Cauchy as in the proof of Theorem 2.3. Since $X$ is complete, $\left\{f x_{n}\right\}$ converges to some $\xi \in X$. Now Theorem 2.4 follows from Theorem 2.3 .

Remark. In case $f=1_{X}$, Theorem 2.4 is reduced to the result of Meir and Keeler [9]. In case $g=f^{2}$, Theorem 2.4 is reduced to the main result of Chung [4].

Corollary 2.5. Let $f$ be a continuous selfmap of a complete metric space $X$ and $g$ be in $C_{f}$. If $g^{N}$ is an $(\varepsilon, \delta)$-f-contraction for some positive integer $N$, then $f$ and $g$ have a unique common fixed point.

Proof. Clearly we have $g^{N} f=f g^{N}$ and $g^{N} X \subset f X$, and hence $g^{N} \in C_{f}$. Applying Theorem 2.3, we have a unique common fixed point $\eta$ of $f$ and $g^{N}$. Then we have $f g \eta=g f \eta=g \eta$ and $g^{N} g \eta=g g^{N} \eta=g \eta$. Hence $g \eta$ is also a common fixed point of $f$ and $g^{N}$. This implies $g \eta=\eta$ because of the uniqueness. Suppose $\eta$ and $\eta^{\prime}$ are common fixed points of $f$ and $g$. Then $g^{N} \eta=\eta=f \eta$ and $g^{N} \eta^{\prime}=\eta^{\prime}=f \eta^{\prime}$. Since $f$ and $g^{N}$ have a unique common fixed point, we have $\eta=\eta^{\prime}$.

Corollary 2.6. If $f$ is a bijective continuous selfmap of a complete metric space $X$, and for any $\varepsilon>0$, there exists $\delta>0$ such that for all $x, y \in X$,

$$
\varepsilon \leqq d(f x, f y)<\varepsilon+\delta \text { implies } d(x, y)<\varepsilon,
$$

then $f$ has a unique fixed point.

Proof. In Theorem 2.4, we set $g=1_{X}$. 
Corollary 2.7. Let $f$ be a continuous selfmap of a complete metric space $X$ and $\left\{g_{\lambda}\right\}_{\lambda \in \Lambda}$ a commuting family of selfmaps in $C_{f}$. If each $g_{\lambda}$ is an $(\varepsilon, \delta)-f$-contraction, then there exists a unique point $\eta \in X$ such that $f \eta=g_{,} \eta=\eta$ for every $\lambda \in A$.

Proof. For each $\lambda, g_{\lambda}$ and $f$ have a unique common fixed point, say $\eta$. For any $\mu \in \Lambda, g_{\lambda}\left(g_{\mu} \eta\right)=g_{\mu}\left(g_{\lambda} \eta\right)=g_{\mu} \eta=g_{\mu}(f \eta)=f\left(g_{\mu} \eta\right)$ implies $g_{\mu} \eta=\eta$ by the uniqueness.

In certain case the continuity of $f$ in Theorem 2.4 can be relaxed to that of some iterate of $f$.

Corollary 2.8. Let $f$ be a selfmap of a complete metric space $X$ such that $f^{k}$ is continuous for some positive $k$. Let $g: f^{k-1} X \rightarrow X$ be a map such that $g f^{k-1} X \subset f^{k} X$ and $g f=f g$ whenever both sides are defined. If $g f^{k-1}$ is an $(\varepsilon, \delta)-f^{k}$-contraction, then $f$ and $g$ have a unique common fixed point.

Proof. By Theorem 2.4, $g f^{k-1}$ and $f^{k}$ have a unique common fixed point $\eta$. From $g f^{k-1}(f \eta)=g\left(f^{k} \eta\right)=g \eta$ and $g f^{k-1}(f \eta)=f\left(g f^{k-1} \eta\right)=f \eta$, we have $f \eta=g \eta$. From $f^{k}(f \eta)=f\left(f^{k} \eta\right)=f \eta$, we know that $f \eta$ is also a common fixed point of $g f^{k-1}$ and $f^{k}$. Therefore, we have $\eta=f \eta=g \eta$. The uniqueness is clear.

Remark. The class of $(\varepsilon, \delta)$-f-contractions contains the classes of selfmaps satisfying $\left(2^{\prime}\right)$ and one of the following conditions:

(8) There exists a map $\phi:[0, \infty) \rightarrow[0, \infty)$ which is upper-semicontinuous from the right such that $\phi(t)<t$ for all $t>0$ and

$$
d(g x, g y)<\phi(d(f x, f y)), \quad f x \neq f y .
$$

(9) There exists a nondecreasing map $\phi:[0, \infty) \rightarrow[0, \infty)$ which is continuous from the right such that $\phi(t)<t$ for all $t>0$ and

$$
d(g x, g y)<\phi(d(f x, f y)), \quad f x \neq f y .
$$

(10) There exists an $\alpha \in[0,1)$ such that

$$
d(g x, g y) \leqq \alpha d(f x, f y) .
$$

Note that $(10) \Rightarrow(9) \Rightarrow(8) \Rightarrow(2) \Rightarrow(3)$ and that (8) and (10) are investigated by Jeong [7] and Jungck [8], and particular types of (9) by Park [10]. Therefore, certain results in [7], [8], [10] are consequences of ours. Note also that for $f=1_{X},(8)$ and (9) reduce to non-linear contractive type conditions of Boyd-Wong [2] and Browder [3], respectively. Avramescu [1] obtained some results for (10) with $g=1_{X}$ and $f$ surjective. We can easily obtain an extended form of a result in [6] with respect to $(\varepsilon, \delta)$-f-contractions.

Finally, we consider compact metric spaces. 
228 Sehie Park and Jong Sook Bae: Extensions of a fixed point theorem of Meir and Keeler

Theorem 2.9. Let $f$ and $g$ be continuous selfmaps of a compact metric space $X$ such that $g \in C_{f}$ and (3) and (2') hold. Then $f$ and $g$ have a unique common fixed point $\eta$ in $X$, and, for each $x_{0}$ in $X$, any f-iteration of $x_{0}$ under $g$ converges to some $\xi \in X$ satisfying $f \xi=\eta$.

Proof. Given $\varepsilon>0$, consider

$$
\inf \{d(f x, f y)-d(g x, g y) \mid \varepsilon \leqq d(f x, f y)\}=\delta(\varepsilon)
$$

Since $X$ is compact, this infimum is achieved for some $(a, b) \in X \times X$ with $d(f a, f b) \geqq \varepsilon$. Since (3) holds, we have $\delta(\varepsilon)>0$. This shows that $g$ is an $(\varepsilon, \delta)$-f-contraction. Therefore, Theorem 2.9 follows from Theorem 2.4 .

Remark. Theorem 2.9 was proved in [10]. For $f=1_{X}$, Theorem 2.9 is reduced to a result of Edelstein [5].

\section{References}

1. Avramescu, C., Theoremes de point fixe pour les applications contractantes et anticontractantes, Manuscripta Math. 6 (1972), 405-411.

2. Boyd, D. W. and Wong, J. S. W., On nonlinear contractions, Proc. Amer. Math. Soc. 20 (1969), 458-464.

3. Browder, F. E., On the convergence of successive approximations for nonlinear functional equations, Indag. $M a^{*} h .30$ (1968), 27-35.

4. Chung, K.-J., On fixed point thcorems of Meir and Keeler, Math. Japonica 23 (1978), 381383.

5. Edelstein, M., On fixed and perioðiic points under contractive mappings, J. London Math. Soc. 37 (1962), 74-79.

6. IsEkI, K., Application of Zamfirescu's fixed point theorem, Math. Sem. Notes 4 (1976), 215216.

7. JeONG, K. S., Extensions of fixed point theorems of Boyd and Wong, M. S. Thesis, Seoul Nat. Univ., 1978.

8. JunGCK, G., Commuting mappings and fixed points, Amer. Math. Monthly, 83 (1976), 261263.

9. MeIR, A. and Keeler, E., A theorem on contraction mappings, J. Math. Anal. Appl. 2 (1969), $526-529$.

10. PARK, S., Fixed points of $f$-contractive maps, Rocky Mountain J. of Math. 8 (1978), 743-750.

Sehie Park

Jong Sook-Bae

Department of Mathematics

Seoul National University

Seoul 151

Korea 